\title{
Treatment of choroidal neovascularisation in age-related macular degeneration with interferon alfa-2a and alfa-2b
}

\author{
Mark C Gillies, John P Sarks, Paul E Beaumont, Alex B Hunyor, David McKay, \\ Margaret Kearns, Peter J McClusky, Shirley H Sarks
}

\begin{abstract}
Forty eight eyes of $\mathbf{4 2}$ patients with choroidal neovascular membranes and age-related macular degeneration who received three different dose regimens of systemic interferon alfa-2 were studied retrospectively. The response to treatment of 41 eyes of the 37 patients who received at least 4 weeks' treatment was analysed with respect to the change in size of the choroidal neovascular membrane and the visual acuity compared with pretreatment levels. The size of the membrane at the end of the course of treatment had decreased in seven $(17 \%)$ eyes overall, not changed in $16(39 \%)$, and increased in $18(44 \%)$. At the end of treatment, the visual acuity had improved in seven $(17 \%)$ eyes, not changed in $27(66 \%)$, and deteriorated in seven $(17 \%)$. With an average follow up of 10 months after treatment, the visual acuity had deteriorated compared with the pretreatment value in 21 out of 41 (51\%) eyes. Vision improved in some fellow eyes with disciform scars. Side effects were common and often severe. The data suggest that one of the major effects of interferon alfa may be to decrease vascular permeability. While further research may identify a place for interferon alfa in the treatment of choroidal neovascularisation, we were unable to demonstrate that the treatment regimens of systemic interferon alfa we used caused a dramatic benefit to patients with exudative age-related macular degeneration.

(Br F Ophthalmol 1993; 77: 759-765)
\end{abstract}

The current treatment of choroidal neovascular membranes, which frequently destroy central vision, is unsatisfactory. Only one quarter of cases in which a choroidal neovascular membrane arises in an eye with age-related macular degeneration can be successfully ablated with a laser, ${ }^{1}$ and at least one half of these eyes suffer recurrences or develop new choroidal neovascular membranes within 5 years. ${ }^{2}$ The Macular Photocoagulation Study Group found that laser ablation of subfoveal choroidal neovascular membranes (that met the eligibility criteria) resulted in marginally but significantly improved visual acuities and scotomas 2 years after treatment, but this was offset against poorer visual acuity in the treated group compared with the untreated group at 3 months. ${ }^{3}$ When subfoveal choroidal neovascular membranes were completely ablated except for the foveal component patients had significantly improved vision 1 year after treatment compared with untreated patients, but of those followed for 3 years $92 \%$ had lost three or more lines of vision. ${ }^{4}$

Remarkable advances over the past decade have identified a number of cytokines that regulate pathological processes such as neoplasia, wound healing, fibrosis, and angiogenesis at the cellular level. ${ }^{5}$ These raise the possibility of novel treatments.

A recent report suggests that systemic interferon alfa-2a treatment may inhibit the growth of choroidal neovascular membranes in eyes with age-related macular degeneration. ${ }^{6}$ Seven patients with well defined subfoveal membranes were treated with systemic interferon alfa-2a for about 6 weeks. Six patients showed resolution of the membrane on treatment, improved acuity, and smaller scotomas. The diameter of the choroidal neovascular membrane was one disc diameter (1 DD) or less in all six eyes in which the membrane regressed.

Systemic interferon alfa- 2 has been used clinically to treat vascular tumours, including pulmonary haemangiomatosis ${ }^{7}$ and haemangioendotheliomas. ${ }^{8}$ The antiangiogenic activity of interferon is not entirely understood. Interferon alfa inhibits vascular endothelial cell proliferation in vitro, possibly through the induction of $2^{\prime}, 5^{\prime}$-oligoadenylate synthetase which leads to the degradation of viral and cellular RNA. ${ }^{9}$

We present herein a retrospective analysis of 48 eyes of 42 patients with age-related macular degeneration who received systemic interferon alfa-2 (either 2a or 2b) treatment for active choroidal neovascular membranes. These patients were treated around the end of 1991 by several groups of Sydney ophthalmologists acting independently and using different protocols. The large number of patients treated no doubt reflects the public awareness of interferon alfa as a treatment for age-related macular degeneration, which was due to a number of reports in the media at that time. The goal of this study was to examine the effects of interferon alfa on the growth and activity of choroidal neovascular membranes in a large group of patients. 


\section{Patients and methods}

\section{PATIENT SELECTION}

Patients with age-related macular degeneration and an angiographically proved choroidal neovascular membrane which involved any part of the foveal avascular zone (except patient 33) and which could not have been treated with laser without destroying central visual acuity were considered for treatment. Three patients (Nos $13,15,36)$ had other conditions affecting their maculae as well as age-related macular degeneration. Patients did not receive treatment if there was a history of any of the conditions shown in Table 1. Patient 33 had already suffered neovascularisation in spite of laser treatment in her other eye and presented with a small area of leakage away from the foveal avascular zone for which she insisted, of her own accord, she receive treatment with interferon alfa. A full blood examination and hepatic enzymes were performed on all patients before entry into the study. A chest $x$ ray and electrocardiograph were included if there was any concern about cardiovascular disease. Eligible patients received treatment after they understood the treatment alternatives, which were observation only or laser treatment of the membrane, and had signed an appropriate informed consent form. The study protocol was approved by the Sydney Eastern Area Health Service Ethics Committee.

\section{PATIENT DATA AND EVALUATION}

Data were collected from four independent groups of investigators, who used either inter-

\section{Table 1 Patient exclusion criteria}

\begin{tabular}{l} 
General debility \\
Anaemia, thrombocytopenia, granulocytopenia \\
Hepatitis, liver failure \\
Psychosis, dementia \\
Uncontrolled cardiovascular disease \\
congestive cardiac failure \\
cardiac arrhythmias \\
ischaemic heart disease \\
\hline
\end{tabular}

Table 2 Patient background data

\begin{tabular}{ll}
\hline Patients & 42 \\
Female & $28(67 \%)$ \\
Mean age (years) & $73 \cdot 8$ \\
Choroidal neovascular membrane in both eyes & 6 \\
Choroidal neovascular membrane in one eye & 36 \\
$\begin{array}{c}\text { Pathology in other eye } \\
\text { disciform scar (previous choroidal }\end{array}$ & 21 \\
$\quad$ neovascularisation) & 11 \\
$\quad \begin{array}{l}\text { drusen } \\
\text { retinal pigment epithelial atrophy }\end{array}$ & 2 \\
$\quad$ enucleation & 1 \\
amblyopia & 1 \\
Mean duration of symptoms (months) & $2 \cdot 9$ (range l \\
& week-1 year) \\
\hline
\end{tabular}

Table 3 Treatment regimens

\begin{tabular}{llll}
\hline Group & $\begin{array}{l}\text { Dose } \\
\left(\times 10^{6} I U\right)\end{array}$ & Frequency & $\begin{array}{l}\text { Total dose } \\
\text { per week } \\
\left(\times 10^{\circ} I U\right)\end{array}$ \\
\hline I & $\begin{array}{l}1(2 \text { doses }) \\
1 \cdot 5(2 \text { doses })\end{array}$ & Three times per week & 9 \\
II & $\begin{array}{l}3 \text { thereafter } \\
3(2 \text { doses })\end{array}$ & Three times per week & 15 \\
III & 5 thereafter & Daily & 21 \\
\hline
\end{tabular}

feron alfa-2a or 2b: MG, JS, SS: interferon alfa-2b, patients 1-17, 19, 21-23; MK, PM: interferon alfa-2b, patients 24-29; DM: interferon alfa-2a, patients 17, 19, 20; $\mathrm{PB}, \mathrm{AH}$ : interferon alfa-2a, patients 30-42. Forty eight eyes of 42 patients were studied. Patient background data are shown in Table 2.

The outcome measures of primary interest were (i) a change in the size of the membrane at the end of treatment, and (ii) change in the Snellen visual acuity immediately after treatment and at the most recent follow up visit when compared with the pretreatment acuity. Each treating ophthalmologist was asked to supply information concerning the nature of the disease in both the eye that warranted treatment (the 'treated eye') and in the fellow eye. The clinical records and pre and post-treatment fundus colour photographs and fluorescein angiograms of all patients were reviewed by a single observer who judged whether the size of the neovascular membrane had decreased, stayed the same, or increased. Visual acuity was regarded as improved or decreased when a change of two lines or more was recorded.

\section{ADMINISTRATION OF INTERFERON}

All groups received interferon alfa- 2 subcutaneously for at least 6 weeks, unless side effects forced treatment to be withdrawn. Three different regimens were employed (Table 3).

\section{INVESTIGATIONS}

Fluorescein angiography was performed within 2 weeks before treatment and 5 days after finishing treatment. A full blood examination and liver function tests were performed fortnightly.

\section{Results}

Table 4 summarises the pathology present, the visual acuity before and after treatment and at the most recent patient visit, and the change in size of the neovascular membrane in each of the 48 eyes of the 42 patients treated. Small foveal neovascular membranes, which were less than $1 \mathrm{DD}$ in size and encroached on the foveal avascular zone, are noted.

Table 5 summarises the change in size of the neovascular membrane at the end of treatment, and the change in Snellen visual acuity compared with the pretreatment level, in patients receiving $9 \times 10^{6}$ IU and $21 \times 10^{6}$ IU interferon alfa-2 weekly, and all patients studied (the latter including those receiving $15 \times 10^{6} \mathrm{IU}$ weekly). Only 41 eyes of 37 patients who received at least 4 weeks' treatment are included, since the efficacy of lesser periods of treatment is questionable. The results in patients with visual acuity of $6 / 18$ or better before treatment and small foveal neovascular membranes are shown separately, since these groups might be expected to show a better response to treatment.

\section{MEMBRANE SIZE}

The size of the membrane at the end of the course of treatment had decreased in seven out of 41 
Table 4 Clinical summary

\begin{tabular}{|c|c|c|c|c|c|c|c|c|c|c|}
\hline \multirow{3}{*}{\multicolumn{2}{|c|}{ Age, sex }} & & & \multicolumn{4}{|c|}{ Acuity } & \multirow[b]{3}{*}{ CNVM size } & \multirow{3}{*}{$\begin{array}{l}\text { Latest acuity } \\
\text { (months post } \\
\text { treatment) }\end{array}$} & \multirow{3}{*}{$\begin{array}{l}\text { Total dose } \\
\left(\times 10^{\circ}\right)\end{array}$} \\
\hline & & \multicolumn{2}{|l|}{ Pathology } & \multicolumn{2}{|c|}{ Treated eye } & \multicolumn{2}{|c|}{ Other eye } & & & \\
\hline & & Treated eye & Other eye & pre & post & pre & post & & & \\
\hline \multicolumn{11}{|c|}{$9 \times 10^{6} \mathrm{IU}$ per week: } \\
\hline 1 & $63, \mathrm{~F}$ & $\begin{array}{l}\text { New vessel } \\
\text { small foveal }\end{array}$ & Drusen & $6 / 18$ & $6 / 12$ & $6 / 6$ & $6 / 6$ & Smaller & $6 / 18(10)$ & 94 \\
\hline $\begin{array}{l}2 \\
3\end{array}$ & $\begin{array}{l}74, \mathrm{~F} \\
84, \mathrm{~F}\end{array}$ & $\begin{array}{l}\text { New vessel } \\
\text { New vessel } \\
\text { small foveal }\end{array}$ & $\begin{array}{l}\text { Disciform } \\
\text { Disciform }\end{array}$ & $\begin{array}{l}3 / 24 \\
6 / 12\end{array}$ & $\begin{array}{l}3 / 60 \\
6 / 12\end{array}$ & $\begin{array}{l}\text { CF } \\
\text { CF }\end{array}$ & $\begin{array}{l}\text { CF } \\
\text { CF }\end{array}$ & $\begin{array}{l}\text { Larger } \\
\text { Larger }\end{array}$ & $\begin{array}{l}2 / 60(16) \\
6 / 60(11)\end{array}$ & $\begin{array}{l}94 \\
94\end{array}$ \\
\hline 4 & $63, \mathrm{~F}$ & $\begin{array}{l}\text { New vessel } \\
\text { small foveal }\end{array}$ & Disciform & $6 / 6$ & $6 / 24$ & CF & CF & Unchanged & $6 / 18(12)$ & 94 \\
\hline 5 & $65, \mathrm{~F}$ & $\begin{array}{l}\text { New vessel } \\
\text { recurrence after } \\
\text { laser }\end{array}$ & Disciform & $6 / 12$ & $6 / 24$ & $6 / 24$ & $6 / 24$ & Larger & $6 / 18(8)$ & 47 \\
\hline 6 & $75, \mathrm{~F}$ & New vessel both eyes & & $6 / 18$ & $6 / 18$ & $6 / 12$ & $6 / 12$ & $\begin{array}{l}\mathbf{R} \text { larger } \\
\text { L unchanged }\end{array}$ & $\begin{array}{l}6 / 18(11) \\
6 / 12\end{array}$ & 47 \\
\hline 7 & $70, \mathrm{~F}$ & $\begin{array}{l}\text { New vessel } \\
\text { small foveal }\end{array}$ & Disciform & $6 / 12$ & $6 / 12$ & HM & HM & Unchanged & $6 / 12(7)$ & 47 \\
\hline 8 & $72, \mathrm{~F}$ & $\begin{array}{l}\text { New vessel both eyes } \\
\text { small foveal } \mathrm{L}\end{array}$ & & $6 / 60$ & $6 / 18$ & $6 / 60$ & $6 / 24$ & $\begin{array}{l}\text { R smaller } \\
\text { L smaller }\end{array}$ & $\begin{array}{l}6 / 60(2) \\
6 / 60\end{array}$ & 47 \\
\hline $\begin{array}{r}9 \\
10\end{array}$ & $\begin{array}{l}72, M \\
76, M\end{array}$ & $\begin{array}{l}\text { New vessel } \\
\text { New vessel both eyes }\end{array}$ & Disciform & $\begin{array}{l}6 / 18 \\
6 / 36\end{array}$ & $\begin{array}{l}\mathrm{HM} \\
6 / 18\end{array}$ & $\begin{array}{l}6 / 18 \\
6 / 9\end{array}$ & $\begin{array}{l}6 / 9 \\
6 / 12\end{array}$ & $\begin{array}{l}\text { Larger } \\
\mathbf{R} \text { unchanged } \\
\text { L unchanged }\end{array}$ & $\begin{array}{l}\operatorname{HM}(12) \\
6 / 18(13) \\
6 / 12\end{array}$ & $\begin{array}{l}47 \\
47\end{array}$ \\
\hline $\begin{array}{l}11 \\
12 \\
13\end{array}$ & $\begin{array}{l}56, M \\
80, F \\
70, F\end{array}$ & $\begin{array}{l}\text { New vessel } \\
\text { New vessel } \\
\text { New vessel } \\
\text { old leuetic } \\
\text { choroiditis }\end{array}$ & $\begin{array}{l}\text { Atrophic } \\
\text { Drusen } \\
\text { Disciform }\end{array}$ & $\begin{array}{l}6 / 24 \\
6 / 36 \\
6 / 9\end{array}$ & $\begin{array}{l}6 / 36 \\
6 / 18 \\
6 / 24\end{array}$ & $\begin{array}{l}6 / 36 \\
6 / 12 \\
6 / 60\end{array}$ & $\begin{array}{l}6 / 36 \\
6 / 9 \\
6 / 60\end{array}$ & $\begin{array}{l}\text { Larger } \\
\text { Unchanged } \\
\text { Unchanged }\end{array}$ & $\begin{array}{l}6 / 60(8) \\
\text { HM (7) } \\
6 / 24(11)\end{array}$ & $\begin{array}{l}47 \\
47 \\
47\end{array}$ \\
\hline $\begin{array}{l}14 \\
15\end{array}$ & $\begin{array}{l}70, M \\
57, M\end{array}$ & $\begin{array}{l}\text { New vessel } \\
\text { New vessel both eyes } \\
\text { old chorioretinitis }\end{array}$ & Drusen & $\begin{array}{l}6 / 12 \\
6 / 60\end{array}$ & $\begin{array}{l}6 / 18 \\
6 / 60\end{array}$ & $\begin{array}{l}6 / 12 \\
6 / 6\end{array}$ & $\begin{array}{l}6 / 12 \\
6 / 6\end{array}$ & $\begin{array}{l}\text { Larger } \\
\text { R larger } \\
\text { L unchanged }\end{array}$ & $\begin{array}{l}6 / 60(9) \\
6 / 60(12) \\
6 / 6\end{array}$ & $\begin{array}{l}47 \\
47\end{array}$ \\
\hline $\begin{array}{l}16 \\
17\end{array}$ & $\begin{array}{l}92, \mathrm{~F} \\
68, \mathrm{~F}\end{array}$ & $\begin{array}{l}\text { New vessel } \\
\text { New vessel } \\
\text { small foveal }\end{array}$ & $\begin{array}{l}\text { Atrophic } \\
\text { Disciform }\end{array}$ & $\begin{array}{l}6 / 60 \\
6 / 24\end{array}$ & $\begin{array}{l}6 / 36 \\
6 / 60\end{array}$ & $\begin{array}{l}\text { CF } \\
\text { CF }\end{array}$ & $\begin{array}{l}\text { CF } \\
\text { CF }\end{array}$ & $\begin{array}{l}\text { Smaller } \\
\text { Larger }\end{array}$ & $\begin{array}{l}C F(8) \\
6 / 60(13)\end{array}$ & $\begin{array}{l}47 \\
47\end{array}$ \\
\hline 18 & $70, F$ & $\begin{array}{l}\text { New vessel } \\
\text { small foveal }\end{array}$ & Disciform & $6 / 18$ & $6 / 18$ & $6 / 60$ & $6 / 24$ & Unchanged & $6 / 60(11)$ & 44 \\
\hline 19 & $76, M$ & $\begin{array}{l}\text { New vessel } \\
\text { small foveal }\end{array}$ & Enucleated & $6 / 60$ & $6 / 60$ & & & Smaller & CF (11) & 38 \\
\hline $\begin{array}{l}20 \\
21 \\
22^{\star}\end{array}$ & $\begin{array}{l}74, \mathrm{M} \\
81, \mathrm{~F} \\
74, \mathrm{M}\end{array}$ & $\begin{array}{l}\text { New vessel } \\
\text { New vessel } \\
\text { New vessel } \\
\text { small foveal }\end{array}$ & $\begin{array}{l}\text { Drusen } \\
\text { Drusen } \\
\text { Disciform }\end{array}$ & $\begin{array}{l}6 / 18 \\
6 / 60 \\
2 / 60\end{array}$ & $\begin{array}{l}6 / 18 \\
6 / 60 \\
2 / 60\end{array}$ & $\begin{array}{l}6 / 6 \\
6 / 9 \\
\text { CF }\end{array}$ & $\begin{array}{l}6 / 6 \\
6 / 9 \\
\mathrm{CF}\end{array}$ & $\begin{array}{l}\text { Larger } \\
\text { Larger } \\
\text { Larger }\end{array}$ & $\begin{array}{l}6 / 60(13) \\
6 / 60(2) \\
\text { CF }(2)\end{array}$ & $\begin{array}{l}35 \\
32 \\
17\end{array}$ \\
\hline $23 \star$ & $80, F$ & $\begin{array}{l}\text { New vessel } \\
\text { small foveal }\end{array}$ & Disciform & $6 / 24$ & $6 / 18$ & CF & $3 / 60$ & Larger & $6 / 24(10)$ & 11 \\
\hline $\begin{array}{l}15 \times 1 \\
24 \\
25 \\
26 \\
27 \\
28 \\
29\end{array}$ & $\begin{array}{l}10^{6}, \mathrm{IUpe} \\
79, \mathrm{~F} \\
81, \mathrm{M} \\
84, \mathrm{~F} \\
62, \mathrm{M} \\
80, \mathrm{M} \\
83, \mathrm{~F}\end{array}$ & $\begin{array}{l}\text { week: } \\
\text { New vessel } \\
\text { New vessel } \\
\text { New vessel } \\
\text { New vessel } \\
\text { New vessel } \\
\text { New vessel }\end{array}$ & $\begin{array}{l}\text { Disciform } \\
\text { Amblyopia } \\
\text { Disciform } \\
\text { Drusen } \\
\text { Drusen } \\
\text { Drusen }\end{array}$ & $\begin{array}{l}6 / 18 \\
6 / 6 \\
6 / 12 \\
6 / 24 \\
\text { CF } \\
6 / 18\end{array}$ & $\begin{array}{l}6 / 24 \\
6 / 6 \\
6 / 18 \\
\text { CF } \\
\text { CF } \\
6 / 9\end{array}$ & $\begin{array}{l}\text { HM } \\
6 / 12 \\
\text { CF } \\
6 / 9 \\
6 / 9 \\
6 / 9\end{array}$ & $\begin{array}{l}\text { HM } \\
6 / 12 \\
\text { CF } \\
6 / 9 \\
6 / 9 \\
6 / 9\end{array}$ & $\begin{array}{l}\text { Larger } \\
\text { Unchanged } \\
\text { Larger } \\
\text { Larger } \\
\text { Smaller } \\
\text { Unchanged }\end{array}$ & $\begin{array}{l}6 / 36(6) \\
6 / 9(12) \\
6 / 18(9) \\
C F(4) \\
C F(13) \\
C F(14)\end{array}$ & $\begin{array}{l}86 \\
86 \\
86 \\
86 \\
86 \\
56\end{array}$ \\
\hline $\begin{array}{l}21 \times 1 \\
30 \\
31\end{array}$ & $\begin{array}{l}10^{6} \mathrm{IU} \text { pe } \\
77, \mathrm{M} \\
67, \mathrm{M}\end{array}$ & $\begin{array}{l}\text { week: } \\
\text { New vessel } \\
\text { New vessel } \\
\text { small foveal }\end{array}$ & $\begin{array}{l}\text { Disciform } \\
\text { Drusen }\end{array}$ & $\begin{array}{l}6 / 12 \\
6 / 24\end{array}$ & $\begin{array}{l}6 / 9 \\
6 / 24\end{array}$ & $\begin{array}{l}6 / 60 \\
6 / 9\end{array}$ & $\begin{array}{l}\text { CF } \\
6 / 6\end{array}$ & $\begin{array}{l}\text { Unchanged } \\
\text { Unchanged }\end{array}$ & $\begin{array}{l}6 / 24(14) \\
\mathrm{CF}(14)\end{array}$ & $\begin{array}{l}126 \\
126\end{array}$ \\
\hline $\begin{array}{l}32 \\
33\end{array}$ & $\begin{array}{l}78, M \\
60, F\end{array}$ & $\begin{array}{l}\text { New vessel } \\
\text { New vessel } \\
\text { hot spot }\end{array}$ & $\begin{array}{l}\text { Drusen } \\
\text { Disciform }\end{array}$ & $\begin{array}{l}6 / 60 \\
6 / 9\end{array}$ & $\begin{array}{l}6 / 60 \\
6 / 9\end{array}$ & $\begin{array}{l}6 / 12 \\
6 / 60\end{array}$ & $\begin{array}{l}6 / 9 \\
6 / 36\end{array}$ & $\begin{array}{l}\text { Unchanged } \\
\text { Smaller }\end{array}$ & $\begin{array}{l}\operatorname{HM}(13) \\
6 / 9(15)\end{array}$ & $\begin{array}{l}126 \\
126\end{array}$ \\
\hline $\begin{array}{l}34 \\
35 \\
36\end{array}$ & $\begin{array}{l}75, F \\
82, F \\
65, F\end{array}$ & $\begin{array}{l}\text { New vessel } \\
\text { New vessel } \\
\text { New vessel } \\
\text { myopic } \\
\text { degeneration, } \\
\text { small foveal }\end{array}$ & $\begin{array}{l}\text { Disciform } \\
\text { Disciform } \\
\text { Detachment }\end{array}$ & $\begin{array}{l}6 / 36 \\
6 / 24 \\
6 / 36\end{array}$ & $\begin{array}{l}6 / 60 \\
6 / 36 \\
6 / 36\end{array}$ & $\begin{array}{l}\text { HM } \\
3 / 60 \\
\text { NPL }\end{array}$ & $\begin{array}{l}\text { CF } \\
3 / 60\end{array}$ & $\begin{array}{l}\text { Larger } \\
\text { Larger } \\
\text { Larger }\end{array}$ & $\begin{array}{l}1 / 60(12) \\
6 / 60(10) \\
6 / 60(11)\end{array}$ & $\begin{array}{l}126 \\
105 \\
103\end{array}$ \\
\hline $\begin{array}{l}37 \\
38 \\
39 \\
40^{\star} \\
41^{\star}\end{array}$ & $\begin{array}{l}70, \mathrm{~F} \\
80, \mathrm{~F} \\
69, \mathrm{~F} \\
68, \mathrm{~F} \\
78, \mathrm{~F}\end{array}$ & $\begin{array}{l}\text { New vessel } \\
\text { New vessel } \\
\text { New vessel } \\
\text { New vessel } \\
\text { New vessel both eyes } \\
\text { both small foveal }\end{array}$ & $\begin{array}{l}\text { Drusen } \\
\text { Disciform } \\
\text { Disciform } \\
\text { Disciform }\end{array}$ & $\begin{array}{l}6 / 18 \\
6 / 18 \\
6 / 18 \\
6 / 24 \\
6 / 24\end{array}$ & $\begin{array}{l}6 / 9 \\
6 / 12 \\
6 / 18 \\
6 / 36 \\
6 / 60\end{array}$ & $\begin{array}{l}\text { 6/6 } \\
\text { HM } \\
6 / 60 \\
6 / 60 \\
6 / 12\end{array}$ & $\begin{array}{l}6 / 9 \\
\text { HM } \\
6 / 18 \\
6 / 60 \\
6 / 24\end{array}$ & $\begin{array}{l}\text { Unchanged } \\
\text { Larger } \\
\text { Unchanged } \\
\text { Larger } \\
\text { R larger } \\
\text { L larger }\end{array}$ & $\begin{array}{l}6 / 9(6) \\
\mathrm{HM}(12) \\
6 / 24(16) \\
2 / 60(12) \\
6 / 60(11) \\
6 / 36\end{array}$ & $\begin{array}{r}103 \\
93 \\
91 \\
69 \\
63\end{array}$ \\
\hline $42^{\star}$ & $79, F$ & New vessel both eyes & & $6 / 36$ & $6 / 18$ & $6 / 12$ & $6 / 12$ & $\begin{array}{l}\mathbf{R} \text { unchanged } \\
\mathrm{L} \text { unchanged }\end{array}$ & $\begin{array}{l}6 / 36(9) \\
6 / 12\end{array}$ & 27 \\
\hline
\end{tabular}

$\star$ Received less than 4 weeks' treatment.

CNVM = choroidal neovascular membrane.

$\mathrm{CF}=$ counting fingers.

$\mathrm{HM}=$ hand movements.
NPL $=$ no perception of light.

(17\%) (Figs 1, 2) eyes overall, not changed in 16 (39\%), and increased in 18 (44\%) (Fig 3). Similar proportions were found both in patients whose visual acuity was $6 / 18$ or better before treatment and patients with a small foveal membrane. No difference was found between patients receiving $21 \times 10^{6}$ IU per week. Of five patients who withdrew before they had been treated for 4 weeks, two eyes had unchanged membranes while five had deteriorated. At least one patient was stable on the higher dose and deteriorated when the dose was reduced (Fig 4). No clear difference was found between patients receiving interferon alfa- $2 \mathrm{a}$ and $2 \mathrm{~b}$; this comparison would in any case be confounded by the different dosages used of the different subtypes.

VISUAL ACUITY

The visual acuity immediately after treatment was improved or unchanged in more patients than was the size of the membrane. Overall, the acuity had improved in seven out of $41(17 \%)$ 'treated' eyes, not changed in $27(66 \%)$, and 
Table 5 Response to treatment

\begin{tabular}{|c|c|c|c|c|c|c|c|c|c|}
\hline & \multicolumn{3}{|c|}{$9 \times 10^{\circ} \mathrm{IU}$ per week } & \multicolumn{3}{|c|}{$21 \times 10^{\circ} \mathrm{IU}$ per week } & \multicolumn{3}{|l|}{ All groups } \\
\hline & \multirow[b]{2}{*}{$\begin{array}{l}\text { Membrane } \\
\text { size }\end{array}$} & \multicolumn{2}{|c|}{ Visual acuity } & \multirow[b]{2}{*}{$\begin{array}{l}\text { Membrane } \\
\text { size }\end{array}$} & \multicolumn{2}{|c|}{ Visual acuity } & \multirow[b]{2}{*}{$\begin{array}{l}\text { Membrane } \\
\text { size }\end{array}$} & \multicolumn{2}{|c|}{ Visual acuity } \\
\hline & & $\begin{array}{l}\text { Post } \\
\text { treatment }\end{array}$ & Latest & & $\begin{array}{l}\text { Post } \\
\text { treatment }\end{array}$ & Latest & & $\begin{array}{l}\text { Post } \\
\text { treatment }\end{array}$ & Latest \\
\hline $\begin{array}{l}\text { All eyes } \\
\text { Improved } \\
\text { Unchanged } \\
\text { Worse }\end{array}$ & $\begin{array}{r}5(20 \%) \\
9(36 \%) \\
11(44 \%)\end{array}$ & $\begin{array}{c}(n=25) \\
5(20 \%) \\
14(56 \%) \\
6(24 \%)\end{array}$ & $\begin{array}{c}1(4 \%) \\
13(52 \%) \\
9(36 \%)\end{array}$ & $\begin{array}{l}1(10 \%) \\
5(50 \%) \\
4(40 \%)\end{array}$ & $\begin{array}{l}(n=10) \\
1(10 \%) \\
9(90 \%) \\
0\end{array}$ & $\begin{array}{l}1(10 \%) \\
2(20 \%) \\
7(70 \%)\end{array}$ & $\begin{array}{r}7(17 \%) \\
16(39 \%) \\
18(44 \%)\end{array}$ & $\begin{array}{r}(n=41) \\
7(17 \%) \\
27(66 \%) \\
7(17 \%)\end{array}$ & $\begin{array}{c}2(5 \%) \\
18(44 \%) \\
21(51 \%)\end{array}$ \\
\hline \multicolumn{5}{|c|}{$\begin{array}{l}\text { Eyes with acuity of } 6 / 18 \text { or better before treatment: } \\
(n=14)\end{array}$} & $(n=5)$ & & & $(\mathrm{n}=23)$ & \\
\hline $\begin{array}{l}\text { Improved } \\
\text { Unchanged } \\
\text { Worse }\end{array}$ & $\begin{array}{l}1(7 \%) \\
7(50 \%) \\
6(43 \%)\end{array}$ & $\begin{array}{c}0 \\
10(71 \%) \\
4(29 \%)\end{array}$ & $\begin{array}{l}0 \\
7(50 \%) \\
7(50 \%)\end{array}$ & $\begin{array}{l}1(20 \%) \\
3(60 \%) \\
1(20 \%)\end{array}$ & $\begin{array}{l}1(20 \%) \\
4(80 \%) \\
0\end{array}$ & $\begin{array}{l}1(20 \%) \\
3(60 \%) \\
0\end{array}$ & $\begin{array}{c}2(9 \%) \\
12(52 \%) \\
9(39 \%)\end{array}$ & $\begin{array}{c}2(9 \%) \\
17(74 \%) \\
4(17 \%)\end{array}$ & $\begin{array}{r}2(9 \%) \\
12(52 \%) \\
9(39 \%)\end{array}$ \\
\hline \multicolumn{10}{|c|}{ Eyes with small foveal neovascular membranes: } \\
\hline $\begin{array}{l}\text { Improved } \\
\text { Unchanged } \\
\text { Worse }\end{array}$ & & & & & & & $\begin{array}{l}3(30 \%) \\
4(40 \%) \\
3(30 \%)\end{array}$ & $\begin{array}{l}1(10 \%) \\
8(80 \%) \\
1(10 \%)\end{array}$ & $\begin{array}{l}0 \\
5(50 \%) \\
5(50 \%)\end{array}$ \\
\hline
\end{tabular}

Figure 1 Fluorescein angiograms of patient 19 . The visual acuity had gradually deteriorated to $6 / 60$ over the preceding 5 months. (A) A subfoveal well defined neovascular membrane was seen 3 days before starting treatment. (B) On the day of finishing treatment. In spite of further extension of the superonasal edge of the membrane, there was substantial regression of the inferotemporal region. The visual acuity remained at 6/60 but had dropped to counting fingers 11 months later.

Figure 2 Fluorescein angiograms of patient 33. A small scotoma was noticed by the patient, who had already lost her central vision in her other eye, 4 weeks previously. (A) Three days before starting

treatment a small area of leakage was seen just above the foveal avascular zone 5 minutes after injection of the dye. Visual acuity was 6/9. (B) Three days after finishing treatment. No leakage was found 5 minutes after injection of the dye. Vision remained 6/9, the scotoma resolved, and the pathology had not recurred 15 months later. $(C)$ The fellow eye before treatment had a large disciform scar with considerable leakage 3 minutes after injection of the dye. (D) The fellow eye after treatment 5 minutes after injection of the dye showing less leakage. Vision had improved from $6 / 60$ to $6 / 36$.

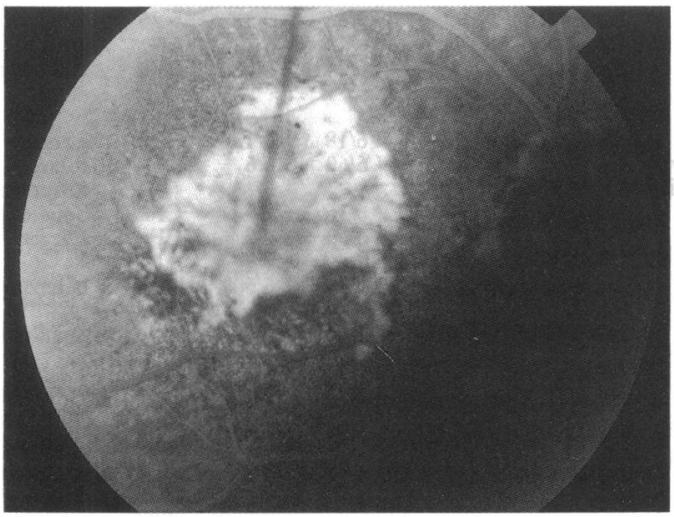

Fig 1A

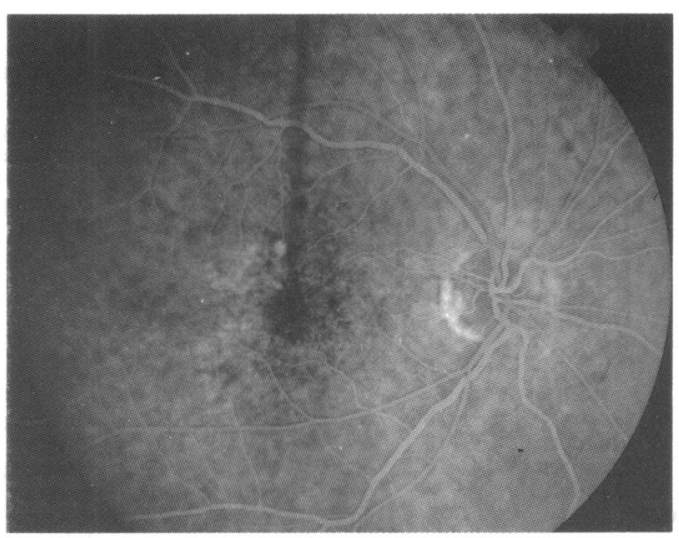

Fig $2 A$

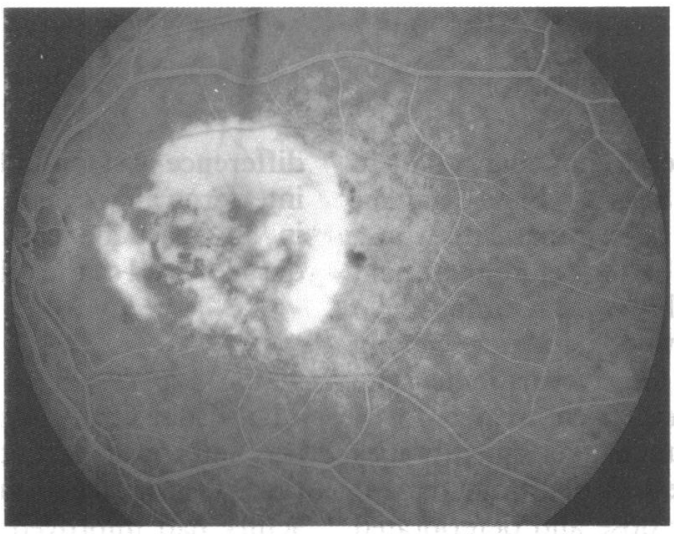

Fig $2 C$

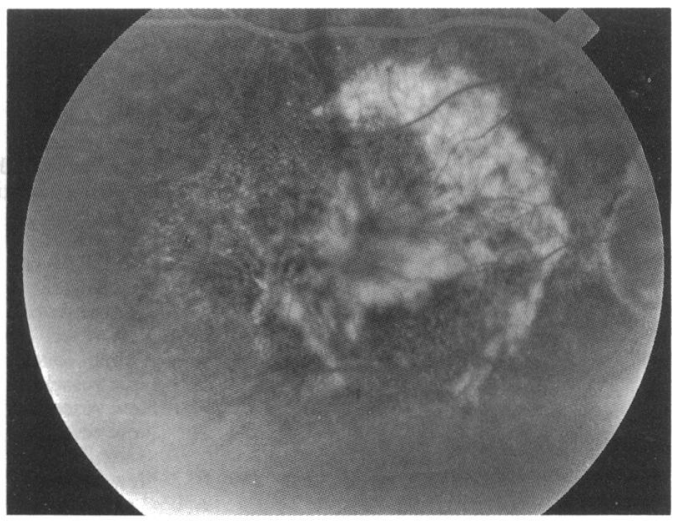

Fig $1 B$

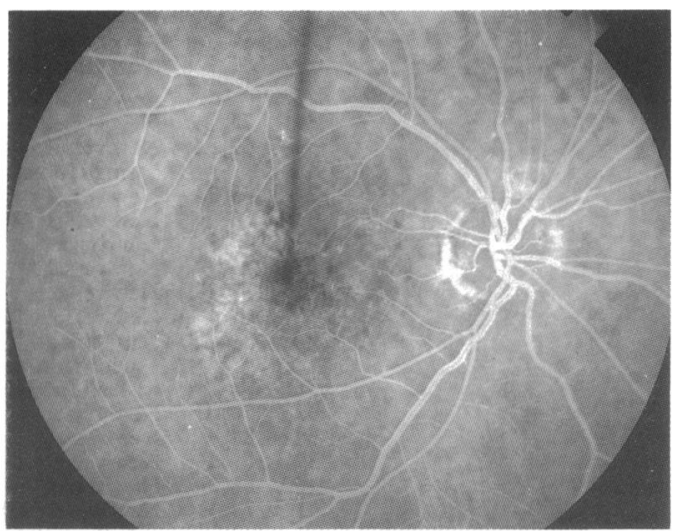

Fig $2 B$

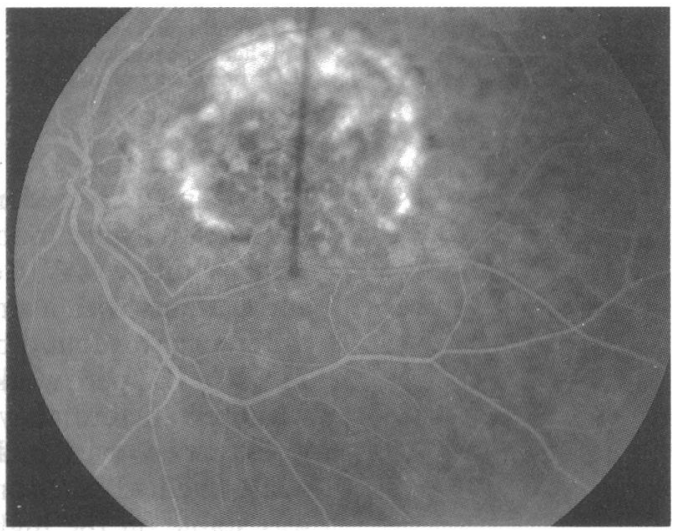

Fig 2D 
Fìgure 3 Fluorescein angiograms of patient 20. The patient had noticed distortion of his central vision for 3 weeks. (A) A small subfoveal well defined choroidal neovascular membrane was seen 5 days before starting treatment. Visual acuity was $6 / 18$.

(B) Three days after finishing treatment. The choroidal neovascular membrane had grown considerably in spite of treatment. While the visual acuity at this stage remained $6 / 18$, it subsequently fell to $6 / 60$.

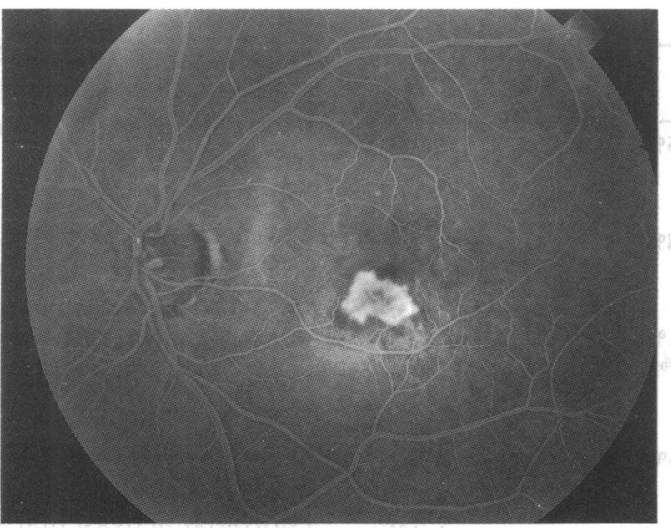

Fig $3 A$

deteriorated in seven (17\%). Again, the groups with better vision or small foveal membranes did not fare significantly better. Ten out of 10 eyes in the patients receiving $21 \times 10^{6} \mathrm{IU}$ per week had better or unchanged visual acuity at the end of treatment, compared with 14 out of $25(56 \%)$ eyes of the patients receiving $9 \times 10^{6} \mathrm{IU}$ per week.

Vision subsequently deteriorated in many but not all eyes. After a mean length of follow up of 10 months (range 2-16), the visual acuity had deteriorated compared with the pretreatment value in 21 out of $41(51 \%)$ eyes overall, nine out of $23(39 \%)$ eyes with the better visual acuity, and five out of 10 eyes with small foveal membranes. Approximately half of the eyes had retained vision within one line of their pretreatment level.

In the 19 fellow eyes where a disciform scar was present, vision improved three lines in one $(5 \%)$,

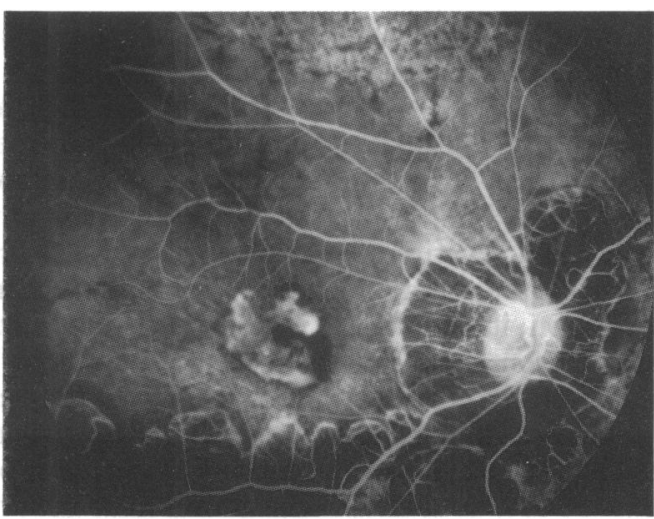

Fig $4 A$

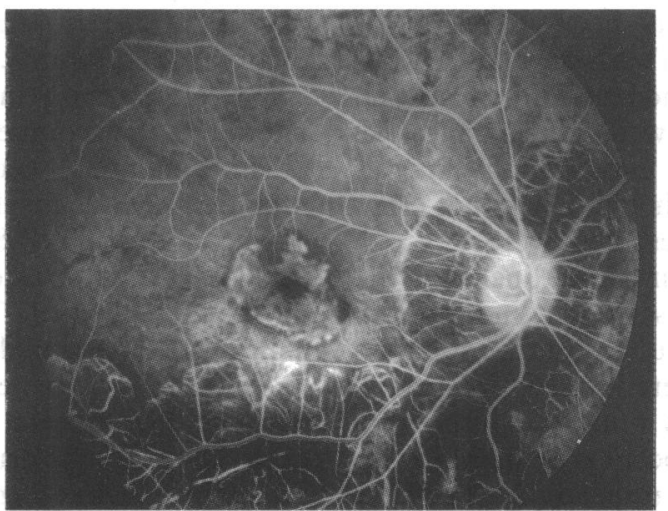

Fig $4 C$

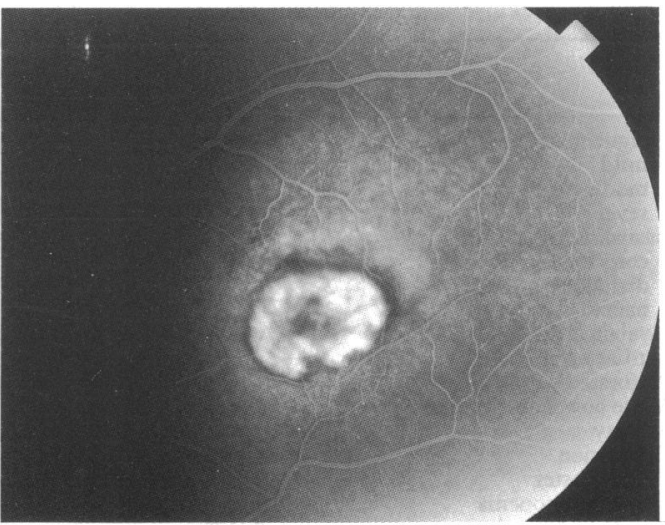

Fig 3B

two lines in two $(11 \%)$, one line in three $(16 \%)$, and remained stable in $13(68 \%)$. Many patients commented that they seemed to be able to 'see through' the scotoma in the eye with the disciform scar. Less leakage was sometimes noted in these eyes with angiography (Fig 2) but this is difficult to quantify and more attention was paid to the eye with the choroidal neovascular membrane.

\section{SIDE EFFECTS}

Table 6 shows side effects reported by patients and conditions developing on treatment in patients receiving $9 \times 10^{6} \mathrm{IU}$ and $21 \times 10^{6} \mathrm{IU}$ interferon alfa- 2 weekly, and all patients studied (the latter including those receiving $15 \times 10^{6} \mathrm{IU}$ weekly). Some side effects, such as mild myalgia,

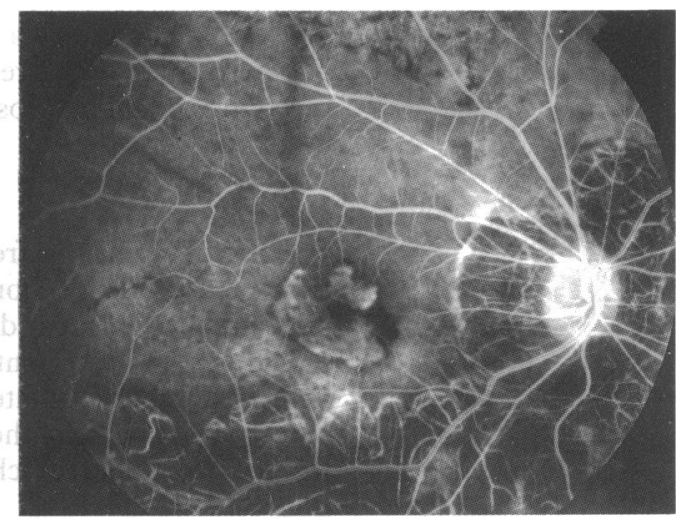

Fig $4 B$

Figure 4 Fluorescein angiograms of patient 36 who had features of age-related as well as myopic macular degeneration. The visual acuity had dropped rapidly to 6/36 over the preceding month. The acuity in the other eye was no perception of light due to a chronic retinal detachment. (A) Three days before treatment there was a well defined foveal neovascular membrane of about $1 \mathrm{DD}$. Extensive myopic chorioretinal atrophy was also seen. (B) After 2 weeks of $21 \times 10^{6} \mathrm{IU}$ per week the mernbrane was fairly stable although there was a small extension at the inferonasal vertical edge. The patient could no longer tolerate the full dose and continued on half the dose of interferon alfa. $(C)$ After 4 weeks of half dose treatment, the membrane had grown from the superonasal area, filling in the nasal notch. When treatment was discontinued, the membrane grew

rapidly and was encircled with laser treatment, resulting in a visual acuity of $6 / 60$. 
Table 6 Side effects and conditions developing during treatment

\begin{tabular}{|c|c|c|c|c|}
\hline & $\begin{array}{l}9 \times 10^{\circ} I U \\
(n=23)\end{array}$ & $\begin{array}{l}21 \times 10^{6} I U \\
(n=13)\end{array}$ & $\begin{array}{l}A l l \\
(n=\end{array}$ & ients \\
\hline \multirow{3}{*}{$\begin{array}{l}\text { Fatigue/malaise } \\
\text { mild } \\
\text { moderate } \\
\text { severe }\end{array}$} & $12(52 \%)$ & $\mathrm{il}(85 \%)$ & & $16(38 \%)$ \\
\hline & 3 & 5 & 10 & \\
\hline & $\begin{array}{l}4 \\
5\end{array}$ & $\begin{array}{l}3 \\
3\end{array}$ & $\begin{array}{l}7 \\
8\end{array}$ & \\
\hline Gastrointestinal & $6(26 \%)$ & $5(38 \%)$ & & $11(26 \%)$ \\
\hline $\begin{array}{l}\text { constipation } \\
\text { diarrhoea } \\
\text { nausea }\end{array}$ & $\begin{array}{l}1 \\
2 \\
3\end{array}$ & $\begin{array}{l}0 \\
2 \\
4\end{array}$ & $\begin{array}{l}1 \\
4 \\
7\end{array}$ & \\
\hline Myalgia/arthralgia & $4(17 \%)$ & $4(31 \%)$ & & $8(19 \%)$ \\
\hline Cardiac arrest & & $1(8 \%)$ & & $1(2 \%)$ \\
\hline Perforated peptic ulcer & & 1 & & \\
\hline Acute bowel obstruction & $1(4 \%)$ & & & 1 \\
\hline Anxiety & & 1 & & $2(5 \%)$ \\
\hline Depression & $2(9 \%)$ & $2(15 \%)$ & & $6(14 \%)$ \\
\hline Herpes zoster & 1 & & & 1 \\
\hline Thrombocytopenia & 1 & & & 1 \\
\hline No adverse reactions & $4(17 \%)$ & 1 & & $4(10 \%)$ \\
\hline Dosage reduced & $4(17 \%)$ & $3(23 \%)$ & & $7(17 \%)$ \\
\hline Treatment discontinued & $2(9 \%)$ & $5(38 \%)$ & & $9(21 \%)$ \\
\hline Received full treatment & $17(74 \%)$ & $5(38 \%)$ & & $26(62 \%)$ \\
\hline
\end{tabular}

may not have been reported by some patients in deference to more serious problems. Generalised fatigue, myalgia, and gastrointestinal complaints were common, especially in the group receiving $21 \times 10^{6} \mathrm{IU}$ per week. It was our impression that the drug was, in general, better tolerated by men.

A number of serious illnesses developed on treatment. One patient suffered a cardiac arrest and died during the fifth week of treatment with $21 \times 10^{6} \mathrm{IU}$ per week. This patient did not have a history of cardiovascular disease or hypertension. A request for a post mortem examination was refused by her relatives. Another patient developed an acute bowel obstruction, another a perforated peptic ulcer. Two patients experienced acute anxiety, six reported depression.

A dose of $9 \times 10^{6} \mathrm{IU}$ per week was better tolerated overall than $21 \times 10^{6}$ IU per week. Seventeen out of $23(74 \%)$ patients receiving the lower dose completed a full course of treatment, compared with only five out of $13(38 \%)$ patients receiving the higher dose $\left(p=0.04, \chi^{2}\right)$.

\section{Discussion}

Bearing in mind the frequency and severity of blindness caused by choroidal neovascularisation in age-related macular degeneration, it is important that new treatments are vigorously sought and thoroughly evaluated. This study provides new data on the use of the cytokine interferon alfa for the treatment of choroidal neovascularisation.

Our results show that systemic interferon alfa treatment does not cause resolution of a significant number of choroidal neovascular membranes in the short term. This is at variance with Fung's report ${ }^{6}$ which found resolution in six out of six patients with small well defined choroidal neovascular membranes encroaching on the foveal avascular zone, although it was not clear whether the patients presented were selected from a large group. Our series included 10 patients who fitted this description, and three of these showed partial resolution, but most either remained the same or deteriorated. Interferon alfa-2a, which was used by Fung, and interferon alfa- $2 b$ differ only at the position 23 amino acid, which is arginine or lysine respectively. They are presumed to have equivalent activity although this has not been established. All the patients receiving the higher dose of interferon in our study received it as interferon alfa- $2 a$. The time from the pretreatment angiogram to starting treatment in our series was up to 2 weeks, so rapid deterioration may have occurred in some eyes after angiography.

The pretreatment visual acuity was maintained in $51 \%$ of eyes at the latest follow up visit with a mean follow up of 10 months. While this result is encouraging, its significance is uncertain. The Macular Photocoagulation Study Group found that 3 months after entry into the study, $52 \%$ of patients with untreated subfoveal choroidal neovascular membranes had lost more than two lines of vision. ${ }^{3}$ In the study of Coscas et al, 20\% of untreated eyes had unchanged or improved visual acuity from 1-4 years after treatment. ${ }^{4}$ All patients in that study had vision between $20 / 100$ to $20 / 1000$ at entry which suggests they may have had longstanding neovascular membranes. Neovascular membranes of recent onset are more likely to deteriorate subsequently, ${ }^{10}$ and the mean duration of symptoms in our patients was only 2.9 months.

The fact that the vision in many eyes deteriorated after treatment suggests that 6 weeks is not long enough. The angiogenic stimulus for choroidal neovascular membranes, which seems to be related to the type and amount of drusen material present, ${ }^{11}$ is unlikely to be altered by interferon alfa treatment. Since drusen usually resolve of their own accord, it may be possible to stabilise choroidal neovascular membranes with interferon alfa until the angiogenic stimulus wanes. In this case it may be necessary to treat the patient for many months with smaller doses of interferon alfa. Alternatively, local administration, such as intravitreal or retrobulbar injection, might allow high sustained local concentrations from a lower dose.

Some of the data in this study suggest that interferon alfa treatment may have reduced the leakage of the neovascular membranes. The small improvement of vision in the fellow eye if a disciform scar were present may well have been due to decreasing vision in the 'treated' eye, but it is also consistent with inhibition by interferon alfa of the persistent leakage from a disciform scar, which was frequently found by Teeters and Bird. ${ }^{12}$ The fact that acuity deteriorated in the 'treated' eye in fewer patients than did the size of the neovascular membrane may also have been due to decreased leakiness of the membrane, although patients may simply have tried harder to read the chart after going through the arduous treatment programme.

Side effects are a major problem of systemic interferon alfa treatment. Fatigue and a flu-like illness commonly occur, while more serious problems include myelosuppression and cardiac and hepatic toxicity. ${ }^{13}$ Some patients in our study developed a life threatening illness during treatment and one died. The severe gastroenterological side effects and mood changes that some of our patients experienced are unusual but can occur with interferon alfa treatment. Previous experience with the drug, which is extensive, has been largely in a younger age group and it should be used in elderly patients with caution. A dose of 
$21 \times 10^{6} \mathrm{IU}$ weekly was intolerable for most of our patients; even $9 \times 10^{6}$ IU weekly was unacceptable in several patients and is unlikely to be tolerated as long term treatment. One of the major challenges in the further evaluation of interferon alfa may be to deliver effective levels of the drug with fewer side effects than we have found.

In this study the patient group was not homogeneous. The absence of a control group precludes any valid statistical analysis. Nevertheless, a number of general conclusions can be drawn on the balance of probabilities from uncontrolled retrospective studies such as this which can be used to direct further research.

The possible benefits of interferon alfa- 2 treatment must be balanced against the risks and side effects. Treatment might be more useful if it could be delivered more effectively for an extended period. Although our regimens seemed to stabilise many patients while they were on treatment, side effects were common and often severe, and many patients relapsed after treatment. We were unable to demonstrate that the treatment regimens of systemic interferon alfa we used caused any dramatic benefit to patients with exudative age-related macular degeneration, although a significant effect may yet be demonstrated by a controlled clinical trial.
Some of the interferon alfa- $2 \mathrm{~b}$ used in this study was donated by $\mathrm{Dr}$ $M$ Rallings, Schering Plough International.

1 Bressler NM, Bressler SB, Gragoudas ES. Clinical characteristics of choroidal neovascular membranes. Arch Ophthalmol 1987; 105: 209-13.

2 Macular Photocoagulation Study Group. Argon laser photocoagulation for neovascular maculopathy. Five year results from rand

3 Macular Photocoagulation Study Group. Laser photocoagulation of subfoveal neovascular membranes in age-related tion of subfoveal neovascular membranes in age-related macular degeneration. Results of a ran

4 Coscas G, Soubrane G, Ramahefasolo C, Fardeau C. Perifoveal laser treatment of subfoveal choroidal new vessels in agerelated macular degeneration. Arch Ophthalmol 1991; 109: $1258-65$.

5 Folkman J, Klagsbrun M. Angiogenic factors. Science 1987; 235: 442-7.

6 Fung WE. Interferon alfa-2a for the treatment of age related macular degeneration. Am f Ophthalmol 1991; 112: 349-50.

7 White CW, Sondheimer HM, Crouch EC, Wilson H, Fan LL. Treatment of pulmonary hemangiomatosis with recombinant interferon alfa 2a. $N$ Engl 7 Med 1989; 320 : $1197-200$.

8 Orchard PJ, Smith CM III, Woods WG, Day DL, Dehner LP, Shapiro $R$. Treatment of haemangioendotheliomas with alpha interferon. Lancet 1989; ii: 565-7.

9 Feldman D, Goldstein AL, Cox DC, Grimley PM. Cultured human endothelial cells treated with recombinant leukocyte A interferon: tuboreticular inclusion formation, antiproliferative effect and $2^{\prime}, 5$ 'oligoadenylate synthetase induction. Lab Invest 1988; 58: 584-9.

10 Teeters VW, Bird AC. The development of neovascularization of senile disciform macular degeneration. Am $\mathcal{F}$ Ophthalmol 1973; 76: 1-18.

11 Sarks SH. Drusen and their relationship to senile macular degeneration. Aust $\mathcal{F}$ Ophthalmol 1980; 8: 117-30.

12 Teeters VW, Bird AC. A clinical study of the vascularity of senile disciform macular degeneration. Am $\mathcal{F}$ Ophthalmol 1973; 75: 53-65.

13 Koeller JM. Biologic response modifiers: the interferon alfa experience. Am $\mathcal{F}$ Hosp Pharm 1989; 46 (Suppl): S11-15. 